\title{
Demands on polarized electron sources by future parity violating experiments
}

\author{
Mark Macrae Dalton* \\ Jefferson Lab \\ E-mail: dalton@jlab.org
}

\begin{abstract}
The next generation of parity-violating electron-scattering experiments at Jefferson Lab will require even greater control of the electron beam under polarization reversal. These experiments require faster transitions between polarization states with smaller associated changes in the beam. In this paper, recent experience at Jefferson Lab is reviewed and the requirements of future experiments are discussed. Potential developments are briefly examined, including the possible use of a Kerr cell to change the helicity of the laser and hence the electron beam.
\end{abstract}

XVth International Workshop on Polarized Sources, Targets, and Polarimetry,

September 9-13, 2013

Charlottesville, Virginia, USA

\footnotetext{
* Speaker.
} 


\section{Introduction}

Parity-violating electron-scattering (PVES) is a very clean way to probe the neutral weakcurrent. The observable of interest is an asymmetry in the electron-scattering cross-section, measured under reversal of the longitudinal polarization of the electron beam. Such a polarization reversal is a parity transformation, which leaves all known forces invariant except the weak interaction. Therefore the asymmetry is a direct probe of the weak interaction.

The technique was originally used to verify the nature of the weak neutral-current [1,2], and more recently has been used to bound the size of the strange quark contribution to the nucleon form factors $[3,4,5,6,7,8]$, verify the existence of a "neutron-skin" in ${ }^{208} \mathrm{~Pb}[9,10]$, and to do precision tests of the Standard Model of particle physics [11, 12]. Asymmetries measured in experiments so far have ranged from $\sim 200$ parts-per-million ( $\mathrm{ppm}$ ) down to $\sim 0.2 \mathrm{ppm}$, depending on the physical process and kinematics.

Production of the polarized electrons and particularly their rapid reversal in the polarized source can produce helicity-correlated beam asymmetries (HCBA), changes in beam properties correlated with the direction of the beam polarization, that lead to measured "false" asymmetries. These require complex corrections and increase the experimental uncertainties. The classic source of false asymmetry is a change in the angle, position or energy of the beam that is correlated with the polarization direction.

Future PVES experiments will need to make measurements with uncertainties smaller, in both absolute and relative terms, than what has been achieved to date. They will therefore be much less able to make corrections and will need to control potential sources of false asymmetries to a higher degree. In addition, for reasons related to the very high luminosity of future experiments, even faster reversal rates will be required.

Recent experience with high precision PVES experiments show that even small HCBA effects can couple to non-linear mechanisms in the accelerator to produce more complex beam asymmetries that may require new techniques to observe. For example, a charge asymmetry hidden in a small portion of the beam phase space, such as the beam halo, is a potential source of false asymmetry if it leads to a proportionally much larger signal in the detectors. This will be discussed in more detail in a later section. A lot of work will be required to meet the specifications of the most ambitious PVES experiments approved to run at Jefferson Lab, the MOLLER [13], SOLID [14], and PREX II [15] experiments.

This paper will present polarized-source related issues encountered in recent PVES experiments. The beam requirements for some major future experiments will be reviewed and potential bottlenecks and solutions will be discussed.

\section{Current technology and limitations}

The polarized electron beam is produced by shining circularly polarized laser light on a doped GaAs photocathode. Reversing the laser polarization reverses the electron polarization. Laser circular-polarization is produced from initial linear-polarization using a Pockels cell (PC) acting as a variable wave plate. A PC is an electro-optic device in which the birefringence is proportional to the applied electric field. The PC is run at the $\frac{1}{4}$-wave voltage of $\sim 2.5 \mathrm{kV}$. The laser circular- 
polarization is then reversed by reversing the voltage on the PC, currently at rates between 30 and $960 \mathrm{~Hz}$.

In the most recent published work on this subject [16], Paschke shows that much of the traditional HCBA arises from the polarization-induced transport-asymmetry (PITA) effect, which is the interaction of a residual linear polarization with the analyzing power of the photocathode, and he describes methods for controlling these effects in the source. In summary, variations of the phase within the laser beam spot caused by birefringence gradients in optical elements, or variations in the analyzing power of the photocathode, will lead to position differences, spot size asymmetries or higher moments of charge distribution asymmetries. These are dealt with using a suite of hard-won techniques to do precision alignment and the use of slow reversals. In addition, divergence in the laser beam through the PC couples phase-shift with position in the beam, and lead to charge asymmetry and position differences that can be tuned to zero with alignment, but also an irreducible spot size asymmetry. Finally, the piezo-electric nature of the PC causes the crystal to steer the beam, like a lens, correlated with the applied voltage. This is minimized by carefully centering the beam on the crystal. The final piece, sometimes called "adiabatic damping", amounts to using reduction of beam emittance during acceleration to also reduce position differences. Achieving the full benefit requires setting up the accelerator optics to minimise coupling between the horizontal, vertical, position and angle dimensions. This could, in principle, achieve a reduction of up to 100 in the measured differences.

The recent drive to have higher polarization reversal frequencies, primarily due to increasing luminosity in experiments, has necessitated faster transitions between polarization states. The large crystals used in the PCs seem to have a characteristic transition speed which depends on the applied voltage. Therefore the transition can be sped up by initially applying a high voltage and then reverting to the required voltage once it is complete. High enough voltages shock the crystal causing the birefringence to oscillate or "ring" for even milliseconds after the excitation. Currently the shortest transitions achieved with a reasonably small crystal response are $50 \mu \mathrm{s}$. Such a dead time is significant to experiments running at the highest frequencies. It may be possible to lessen the shock by shaping the initial high voltage pulse and to actively damp any oscillations with a counteracting signal, but there is a sense that the intrinsic limit of the PC is near.

\subsection{Recent Qweak Experience}

These techniques were recently applied to the setup of the Qweak experiment [11] and lead to the smallest HCBA effects directly measured off the photo-cathode in a physics experiment that we are aware of. However, the beam emittance reduction did not significantly reduce beam differences at the experimental target, in this case. The unprecedented precision of this experiment and the unusually high beam space charge has revealed a new type of beam difference that while not fully understood at the moment, will be a significant source of uncertainty and correction for the experiment and must be considered for future, open-geometry experiments.

Qweak is an experiment that ran over two years at Jefferson Lab to measure the weak charge of the proton. It used a toroidal spectrometer with open geometry to collect elastically scattered electrons from a $1.16 \mathrm{GeV}$ beam with an average scattering angle of $\sim 7.8^{\circ}$. The measured asymmetry is about $200 \mathrm{ppb}$. The $\sim 180 \mu \mathrm{A}$ beam and $35 \mathrm{~cm}$ long liquid hydrogen target lead to a rate of $6 \mathrm{GHz}$ in the detectors. 
The Qweak experiment had, at times, a large amount of beam halo which, using various detectors along the beamline, was determined to have a large helicity-correlated charge-asymmetry. In addition, ancillary detectors in the shielded detector hut show that there is a "beamline" background with asymmetry up to $50 \mathrm{ppm}$, and studies done closing some of the collimator openings show that the main detectors get $\sim 0.2 \%$ of their signal from this background. It is believed that the beamline background is generated by interaction of the halo with apertures in the beamline, particularly a narrow, water-cooled, tungsten collimator shortly after the target, and finds its way to the detectors through the relatively open geometry of the experiment. The background asymmetry arises in the charge-asymmetry of the halo.

The exact mechanism for the production of the large halo and the large charge asymmetry therein, is not currently clear to the author, but this is the subject of continued investigation. It is suspected that it is related to the high beam current, which may have caused beam loading in the warm beam elements of the injector used to bunch the beam. Such high beam currents will not be available for future experiments, but changes to the beam timing structure for future "four-hall" operations could result in similarly high space-charge in future experiments.

\section{Requirements of future experiments}

In terms of beam requirements, the MOLLER experiment is the most challenging future parity violation experiment approved to run at Jefferson Lab. It is another open geometry experiment, designed to measure the weak charge of the electron through Møller scattering at $11 \mathrm{GeV}$. With an exceptionally small scattering angle, $0.29^{\circ}<\theta_{\text {lab }}<0.97^{\circ}$, it will be highly sensitive to beam motions. The specifications call for cumulative helicity-correlated position differences of $<0.5$ $\mathrm{nm}$, angle differences of $<0.05 \mathrm{nrad}$, and a laser spot size difference $<0.01 \%$. With a very small physics asymmetry $\sim 35$ parts-per-billion (ppb), and an intended uncertainty of $\sim 0.73 \mathrm{ppb}$ or $2 \%$, it will be very sensitive to potential false asymmetries. The $80 \mu \mathrm{A}$ beam and $1.5 \mathrm{~m}$ long liquid hydrogen target lead to high luminosity that then requires polarization reversal rates of $\sim 2 \mathrm{kHz}$ or more.

As such, it will push the existing achievements from all directions. The specifications presume that is understood how each type of beam asymmetry affects the physics measurements and the coupling between them. As Qweak found, having beam differences of any kind in the injector is dangerous in that the complex non-linear nature of the accelerator can convert them into a form that is not easily measured and whose effect is not easily understood.

\section{Potential Solutions}

One potential solution to irreducible HCBA coming from the photocathode is to apply a canceling correction. Such a feedback strategy already works well with the overall charge of the beam and is typically implemented on the timescale of a few minutes.

This approach has significant limitations. It can only be applied to effects that can be measured and controlled, thus it could not currently be applied to beam spot size asymmetries, for example, since they cannot be continuously monitored or dynamically controlled. This would require significant $R \& D$ and seems unlikely for the next generation of experiments. For position or angle 
differences, it is possible to use air core magnets in the low energy injector region with fast switching supplies to apply an individual beam correction for each polarization state, and this was done during the latter parts of Qweak experiment. In practice, frequent recalibration of the response of the of these devices was required and, due to the beam optics of the injector and their placement, they were somewhat degenerate in some dimensions. This is a strategy of last resort since there is a concern that any measured HCBA might be accompanied by unmeasurable other effects which would not also be corrected. Even worse, a polarization dependent correction might itself introduce other undesirable effects on the beam.

A recent example of where feedback can have a masking effect of beam asymmetries is beam interception on apertures in the injector. During Qweak, position differences coupled with "clipping" on the injector apertures resulted in a polarization dependent clipping and hence a charge asymmetry. The charge feedback mechanism forced the charge asymmetry at the experiment to always be small, by inducing an initial charge asymmetry at the photo-cathode that was sometimes as large as $200 \mathrm{ppm}$. Differential clipping on apertures presumably must cause the distribution within the two states to be different. For the Qweak experiment, the primary symptom in the experiment was a large width of the charge asymmetry distribution, due to the inherent fluctuations of how much beam was intersected by the apertures. Resteering of the beam was required regularly due to drifting of the front end elements.

\subsection{Kerr Cell}

Another potential solution is to use a new technology, namely a Kerr cell, to reverse the laser polarization. A Kerr cell is cell containing a material demonstrating the Kerr effect, with an applied electric field through which light is propagated. The Kerr effect is a change in the refractive index of a material in proportion to the square of an applied electric field. Such an effect is much weaker than the Pockels effect and thus only centrosymmetric materials (gases, liquids, and certain crystals), which do not demonstrate a Pockels effect, are suitable. A liquid Kerr cell might be able to overcome a number of limitations of the PC, since it should admit a birefringence change more quickly, while birefringence gradients from crystal defects and strains would be absent, as would crystal lensing effects.

The practical issues with a Kerr cell are based on four of the defining features of the Kerr effect: it is very weak, non-linear, independent of the sign of the electric field, and it requires a transverse electric field. The weakness of the effect leads to difficulty in designing the cell, since achieving a $\frac{1}{4}$-wave birefringence will require high voltages, close electrode spacing, long cells and exotic materials such as nitrobenzene or liquid crystals.

Since light is itself a transverse electric field, it induces the Kerr Effect in the material through which it is traveling, even without an external electric field. The electric field of the laser is parallel to it's polarization, so the induced birefringence does not cause a rotation of the light. However, the refractive index of the material, as seen by the laser beam, will vary from the center to the edge, acting as a lens and leading to self focussing of the laser beam which depends on the laser power. Thus a charge asymmetry that is incident on the Kerr cell will induce a beam spot size asymmetry. This can be reduced by shortening the cell (and therefore increasing the high voltage.)

The Kerr effect has no dependence on the sign of the electric field. Thus reversing the laser circular-polarization is more difficult than in the PC case. The easiest way is likely to be using 
a single cell with different voltages for each state. The cell can be run at $\frac{1}{4}$-wave and $\frac{3}{4}$-wave voltages, or similarly it could be run at 0 and $\frac{1}{2}$-wave voltages and followed with a quarter wave plate. Another option is to have a separate set of electrodes at right angles. This is not possible in a single Kerr cell, since the presence of additional electrodes will distort the field in the cell, and would require two cells in series. Either of these two scenarios introduces an explicit difference between the two states which would to require very careful setup to prevent beam asymmetries. One could imagine combining two cells and two voltages to make each state in two different ways to try and cancel such effects.

In a Kerr cell, the symmetry is provided by the applied electric field rather than a crystal structure. Since the effect is quadratic with field, uniformity is very important. The literature on Kerr cells is scarce, but at least one cell, in this case using nitrobenzene, has seen a non-uniform electric field develop between the two parallel plates, apparently due to charge screening effects in the medium [17]. The field appeared to drop sharply at the plates but became remarkably flat toward the center of the cell. The large birefringence gradient would make such a cell useless, particularly if different states had different voltages or electrode angles. It could perhaps be dealt with by widening the cell and attempting to use only the flat area in the center.

The prospect of using a Kerr cell for PVES experiments, while tantalizing, presents many challenges. Continued research is necessary to study to what extent these can be overcome to yield a viable alternative to the ubiquitous PC.

\section{Summary}

In summary, control of the electron beam parameters under polarization reversal remains a challenge for PVES experiments as increased precision makes existing achievements for traditional beam asymmetries inadequate and new classes of effects become important. Higher moment effects, which cannot generally be measured continuously will become significant. Efforts should focus on continuing to diminish beam differences at their source while simultaneously developing correction mechanisms in case they are necessary. Kerr cells could be useful a way to reduce the production of beam asymmetries but there are significant potential issues and development is required.

\section{Acknowledgements}

I must specifically acknowledge Kent Paschke, Manolis Kargiantoulakis, and Gordon Cates from University of Virginia and Matt Poelker, Joe Grames, and John Hansknecht from the Electron Gun Group at Jefferson Lab, all of whom I had the pleasure of working with and made great contributions to the electron source for use in parity violation experiments. Many others have also contributed to these efforts.

\section{References}

[1] C. Prescott, W. Atwood, R. L. Cottrell, H. DeStaebler, E. L. Garwin, et al., Phys.Lett. B77, 347-352 (1978). 
[2] C. Prescott, W. Atwood, R. L. Cottrell, H. DeStaebler, E. L. Garwin, et al., Phys.Lett. B84, 524 (1979).

[3] K. Aniol, et al., Phys.Rev. C69, 065501 (2004).

[4] F. E. Maas, et al., Phys. Rev. Lett. 93, 022002 (2004), URL http://Iink.aps.org/doi/10.1103/PhysRevLett.93.022002.

[5] D. S. Armstrong, et al., Phys. Rev. Lett. 95, 092001 (2005), URL http://link.aps.org/doi/10.1103/PhysRevLett.95.092001.

[6] K. Aniol, et al., Phys.Rev.Lett. 96, 022003 (2006).

[7] A. Acha, et al., Phys.Rev.Lett. 98, 032301 (2007).

[8] Z. Ahmed, et al., Phys.Rev.Lett. 108, 102001 (2012).

[9] S. Abrahamyan, Z. Ahmed, H. Albataineh, K. Aniol, D. Armstrong, et al., Phys.Rev.Lett. 108, 112502 (2012).

[10] C. Horowitz, Z. Ahmed, C. Jen, A. Rakhman, P. Souder, et al., Phys.Rev. C85, 032501 (2012).

[11] D. Androic, et al., Phys.Rev.Lett. 111, 141803 (2013).

[12] D. Wang, et al., Nature 506, 67-70 (2014).

[13] K. Kumar, et al. (2008), URL http://www.jlab.org/exp_prog/proposals/09/PR12-09-005.pdf.

[14] P. Souder, et al. (2009), URL http://www.jlab.org/exp_prog/proposals/10/PR12-10-007.pdf.

[15] K. Paschke, et al. (2011), URL http: //www.jlab.org/exp_prog/proposals/11/PR12-11-101.pdf.

[16] K. Paschke, Eur.Phys.J. A32, 549-553 (2007).

[17] A. W. Knudsen, Am. J. Phys. 43, 888-894 (1975). 